\title{
Seminar on Pseudo-Hirschsprung's Disease and Related Disorders
}

This Seminar and the two that follow were held at the Combined Meeting of the British Association of Paediatric Surgeons and the Surgical Section of the American Academy of Pediatrics, Edinburgh, July 27-30, 1965.

Moderators : J. F. R. Bentley (Glasgow), H. H. Nixon (London), Th. Ehrenpreis (Stockholm), and B. SPENCER (Minneapolis).

Discussants: J. Lister (Sheffield), B. Duhamel (Paris), R. Pagès (Paris), and A. Katz (Cape Town).

\section{'Pseudo-Hirschsprung's Disease'}

TH. EHRENPREIS

Last year at the B.A.P.S. meeting in Rotterdam, I read a paper on Pseudo-Hirschsprung's Disease, presenting a patient with essentially the same clinical and radiological picture as in Hirschsprung's disease. However, no histological abnormality was found in the narrow segment resected. A few similar cases had been published earlier and few further cases were reported in the following discussion.

Our discussion on pseudo-Hirschsprung's disease today has a much wider scope. It is doubtful whether the title of this seminar is quite correct. Something like 'Hirschsprung's disease and allied disorders' might have been more appropriate. The main thing to remember is that the various disease patterns are essentially determined by their underlying pathology, irrespective of what we choose to call them. It would obviously be of great value if some rough agreements could be reached with regard to terminology which at present is rather confused and confusing. It might be helpful before entering into the discussion to consider the various disorders in question and the different names they have been given. In Table I you will find all disorders I know of in this field and some I don't know much about. The various disorders have been grouped according to the underlying pathology, as far as it is known at the present time. Common synonyms for their denominations are listed. The names given in italic letters are those mostly used.

The first group comprises disorders caused by abnormality of ganglion cells: Hirschsprung's disease (including its short- and long-segment varieties), the South-American Chagas' disease, and two conditions that I hope to learn more about today: hypoganglionosis and immaturity of ganglion cells.

The second column deals with disorders without abnormality of intramural ganglion cells. So far we don't know the underlying pathology. Idiopathic megacolon and its synonyms are the big subdivision in this group. I don't like the use of the term pseudoHirschsprung as a synonym for idiopathic megacolon, as I

TABLE I

Hirschsprung's Disease and Allied Disorders

\begin{tabular}{|c|c|c|}
\hline \multirow{2}{*}{ With Abnormality of Ganglion Cells } & \multicolumn{2}{|c|}{ Without Abnormality of Ganglion Cells } \\
\hline & Aetiology Obscure & Clear Aetiology \\
\hline $\begin{array}{l}\text { Hirschsprung's disease } \\
\text { Congenital megacolon } \\
\text { Congenital aganglionosis } \\
\text { Chagas' disease } \\
\text { Acquired megacolon } \\
\text { Aperistalsis } \\
\text { Hypoganglionosis (Bentley) } \\
\text { Immaturity of Ganglion cells (Spencer) }\end{array}$ & $\begin{array}{l}\text { Idiopathic megacolon } \\
\text { Functional megacolon } \\
\text { Psychogenic megacolon } \\
\text { Megarectum } \\
\text { Colonic inertia } \\
\text { Chronic constipation } \\
\text { (Pseudo-Hirschsprung, Ravitch) } \\
\text { Pseudo-Hirschsprung } \\
\text { (Ehrenpreis) } \\
\text { Segmental dilatation of the colon } \\
\text { (Swenson and Rathauser) } \\
\text { Achalasia of distal rectal segment } \\
\text { (Davidson and Bauer) }\end{array}$ & $\begin{array}{l}\text { Symptomatic megacolon } \\
\text { Secondary megacolon } \\
\text { (anal stricture, myxoedema, } \\
\text { cerebral atrophy) }\end{array}$ \\
\hline
\end{tabular}


need this term for the denomination of the more rare condition I described last year. Segmental dilatation of the colon, again, should be distinguished both from idiopathic megacolon and pseudo-Hirschsprung's disease. Achalasia of a distal rectal segment was described by Davidson and Bauer in 1958 as a specific disease entity without abnormality of ganglion cells and without a distal narrow segment. On motility studies, however, the distal rectal segment behaved like an aganglionic area.

Symptomatic megacolon, finally, is a well-defined group with clear aetiology and needs no further comment.

The most interesting groups at the present time are those in the second column. I would like to end this short introductory survey by quoting from a recent paper of Bentley's: 'When simple histologic examination reveals no abnormality in the wall of the bowel, subtle intrinsic factors (possibly inborn) might still exist to impair the reflex of defecation. As knowledge of neuro-chemistry and smooth muscle activity increases, more sophisticated technics may develop to study the tissue excised from the rectum.'

\section{Posterior Excisional Ano-rectal Myotomy in Management of Chronic Faecal Accumulation}

\section{J. F. R. BENTLEY}

In infants and children, dilatation of the rectum by intractable accumulation of faeces may originate in several ways, but these are not always easy to distinguish by the history or the physical and radiological signs. Some of the patients may have ultra-short segment Hirschsprung's disease, and histological examination is then required to identify the abnormal innervation of the wall of the distal bowel. The minor operation of posterior excisional ano-rectal myotomy (PEARM) not only provides a representative biopsy to reveal such abnormalities, but it can also assist palliation of the symptoms. Such palliation is particularly evident in Hirschsprung's disease where the aganglionic segment is confined to the distal third of the rectum.

The operation has been performed on 22 patients who have been subsequently observed for 2 to 9 years. 10 had the intrinsic neurohistology of Hirschsprung's disease and a further 5 also had abnormalities of the intrinsic nerves of debatable aetiology that resembled those of the transitional zone of Hirschsprung's disease. In the remaining 7 the bowel histology was normal.

The operation: A complete longitudinal section of the posterior aspect of the anal canal and distal rectum is excised. The strip is about $5 \mathrm{~mm}$. wide and up to $10 \mathrm{~cm}$. long. The resultant myotomy relaxes the internal anal sphincter and the muscular wall of the lower rectum, as only the ano-rectal mucosa and sub-mucosa are repaired by suture. Where symptoms are not unduly acute the bowel is emptied and 'sterilized' before operation. Tetracycline is given for a week after the procedure. The patient is placed in the lithotomy-Trendelenburg position under general anaesthesia and $5 \mathrm{ml} .1: 125,000$ aqueous noradrenaline are infiltrated into the posterior ano-rectal wall to assist haemostasis. A head-light and a bivalve anal speculum are used to illuminate and expose the operative field. A gauze pack is lightly inserted in the upper lumen, and a small triangular flap of anal skin is reflected forward to expose the posterior lower border of the internal anal sphincter. (The pallor of this muscle is less evident in infancy.) The interval between the internal and external anal sphincters is defined, and a strip of bowel wall about $5 \mathrm{~mm}$. wide is cut with scissors. (One blade is placed between the sphincters and the other lies in the lumen of the bowel.) To avoid damage to the peritoneum the strip is cut towards the hollow of the sacrum. Excision is facilitated by traction on the specimen, but it is not severed until the first stitch is in place. The suture is of 000 chromic cat-gut threaded on a $5 / 8$ circle 16 or $25 \mathrm{~mm}$. needle. The ends of the catgut are knotted together, and the first stitch is locked in position by passing the needle through the loop. Traction on the suture assists the insertion of further stitches as far as the anal verge, but a small post-anal wound is left open for drainage (Fig. 1 and 2). The intraluminal pack is replaced with oxycellulose gauze, and flat gauze dressings impregnated with $1 \%$ cetrimide are applied to the anus and changed daily. A spontaneous and relatively painless bowel action often occurs on the second or third post-operative day.

The operation has proved safe and haemorrhage has not been a complication. 2 patients had transient postoperative pyrexia. When the procedure is restricted to young patients with chronic constipation and the external anal sphincter is not damaged, permanent faecal incontinence is unlikely, but as transient post-operative anal incontinence may last several weeks discrimination is required in the selection of patients. A posterior scar is left in the lower rectum which can complicate subsequent resection and anastomosis.

The 22 young patients included an infant aged 6 weeks. Most suffered from chronic constipation and accumulation of faeces in the rectum that failed to respond to prolonged medicinal treatment, but none had anal stenosis or an evident lesion of the spinal cord. The microscopical appearances of haematoxylin and eosin sections from the rectal biopsies fell into three groups: (a) Normal histology, 7 cases ; (b) Hirschsprung's disease, 10 cases; and (c) abnormal myenteric nerve with a few ganglion cells, 5 cases.

Group (a). Normal histology. There were 3 girls and 4 boys between $3 \frac{1}{2}$ and 8 years of age. 6 developed severe constipation in the first or second year of life, and in the seventh the symptoms started at the age of 4 years. 2 boys eventually died of renal failure with bilateral hydronephrosis, megaureters, and megacystis: both had received psychiatric care for enuresis and faecal retention with soiling. One psychologically deprived girl with similar symptoms continues to have enuresis, despite wedge resection of the bladder neck, excision of a large bladder diverticulum, and long-term antibacterial medication for infection of the urinary tract, but she empties her bowels daily without intercurrent soiling. Two boys and a girl have slowly lost their symptoms, but one girl remains unchanged. 

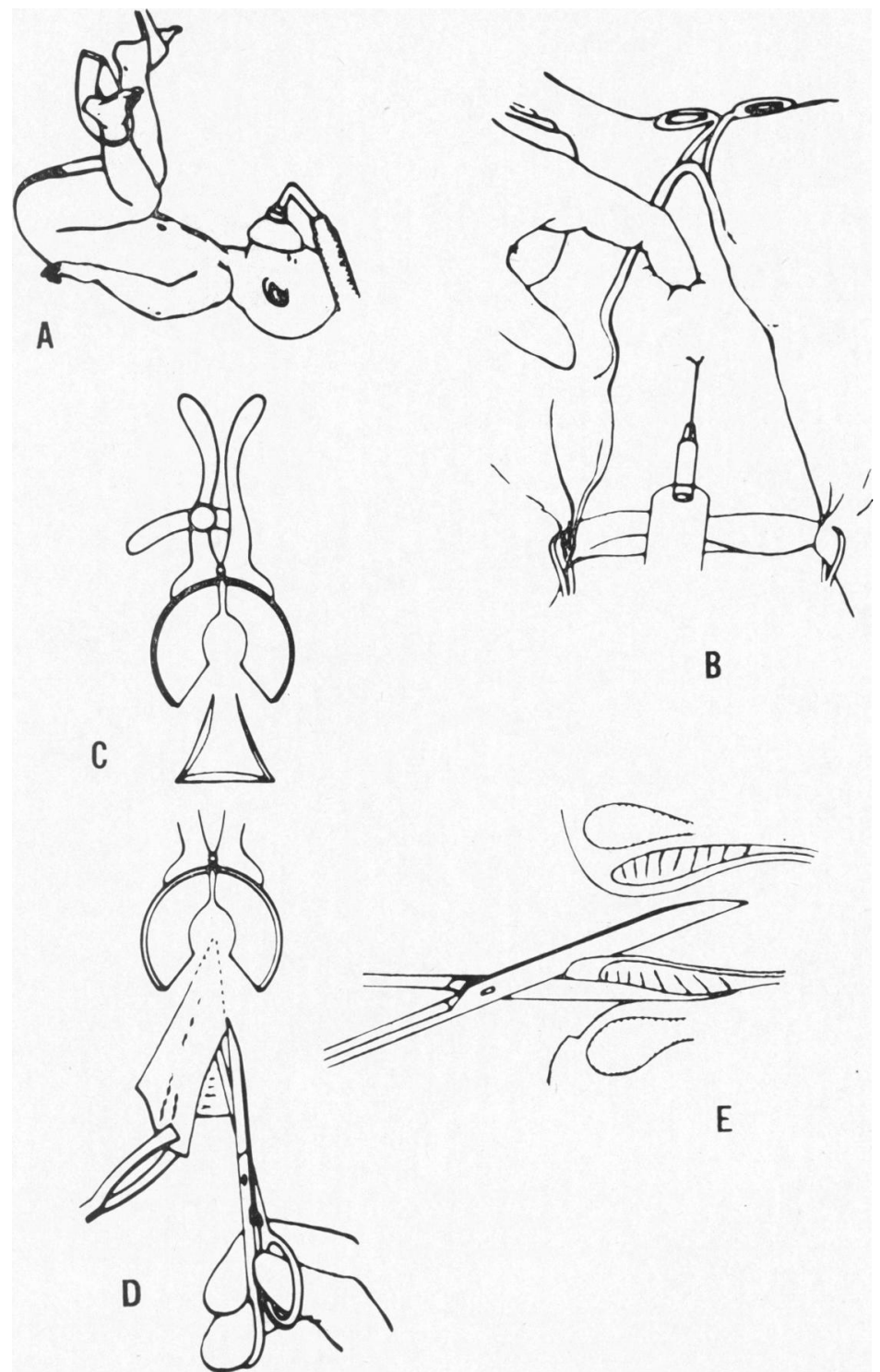

Fig. 1.-Posterior excisional ano-rectal myotomy. A. Lithotomy-Trendelenburg position. B. Infiltration of ano-rectal wall with 1 : 125,000 aqueous noradrenaline. C. Post-anal triangular skin flap cut after insertion of speculum. D. Traction assists in cutting the specimen. E. One blade of the scissors is placed between the internal and external anal sphincter and the other lies in the lumen of the bowel.

Group (b). Hirschsprung's disease. There were 9 boys and 1 girl in this group, their ages ranged from 6 weeks to 8 years. 6 of the boys have been treated without major operation, 3 of them have spontaneous daily bowel evacuation, but the other 3 continue to use suppositories or a laxative. In 4 patients the symptoms dated from infancy, in 1 they started in the second year, and in 1 at the age of 4 years. One boy suffered from chronic faecal soiling. In the nine years following operation one 17-year-old boy has had no disability except for an acute episode of constipation that was precipitated by an attempt to curtail his daily dose of paraffin emulsion. Another boy with a transverse colostomy that had been established for obstructive symptoms had this closed after a 'PEARM' operation at the age of 7 years. He remained free of symptoms with 


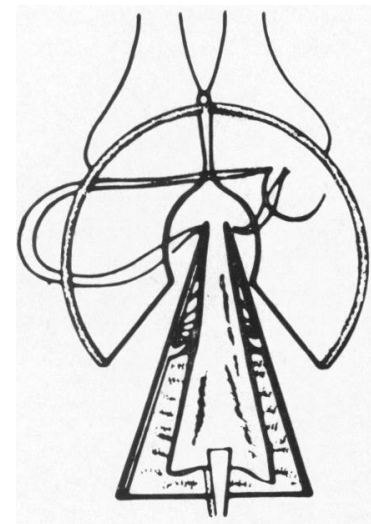

A

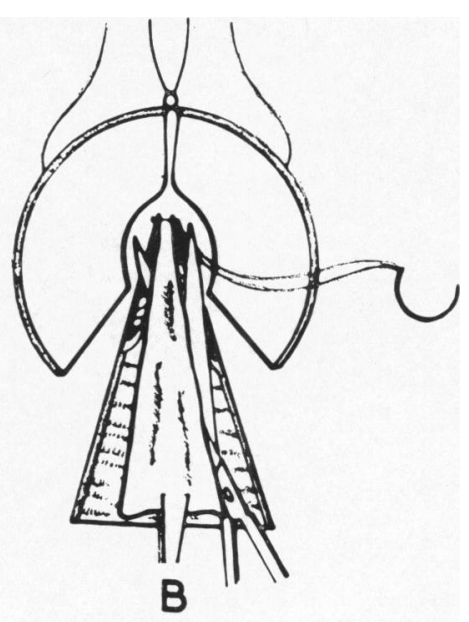

B

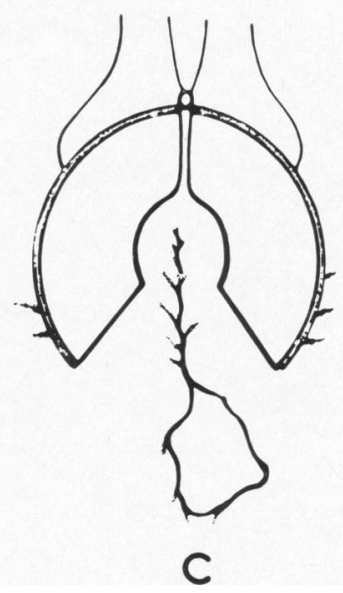

FIG. 2.-Posterior excisional ano-rectal myotomy (continued). A. Traction on the specimen is maintained while the first stitch is locked in position by passing the needle back through the knotted loop. B. The specimen is excised. C. The mucosa and submucosa are approximated by continuous suture. A small post-anal area is left open for drainage.

decreasing distension of the abdomen for the next two months, but since then this former chronic attender has been untraceable for 3 years. Another boy had a colostomy established in infancy, but this was closed after a 'PEARM' operation. He was subject to attacks of faecal impaction for some months thereafter, but at the age of 2 years he evacuates well with the aid of suppositories, one being inserted every third day. The remaining boys have spontaneous bowel actions: they have been followed for 2, 3, and 6 years. The last-mentioned infant had a 'PEARM' operation at the age of 2 months while suffering from obstruction. The distal myenteric plexus was aganglionic with unmyelinated nerve fibres. He made an excellent recovery apart from an early faecal impaction that responded to anal dilatation and removal of faeces, and he remains symptom free. Radiological examination shows no residual abnormality of the colon or rectum.

Of these patients, 4 have been treated by Swenson's operation : 3 of them were early in the series and included the girl. She had a transverse colostomy established for obstruction in early infancy, and the 'PEARM' operation was performed to obtain histological confirmation of the diagnosis, before a successful resection for extensive aganglionosis of the colon and rectum. The 'PEARM' operation is no longer used exclusively for diagnosis in this way when a major resection can be anticipated, as disruption of the anastomosis led to the death of the 2 boys. At that time the diagnosis of Hirschsprung's disease was accepted as the indication for Swenson's operation, but in these boys extensive rectal resection removed negligible aganglionic bowel. In one the ultra- 
short aganglionic segment lay entirely below the level of the anastomosis, and in the other only $1 \mathrm{~cm}$. of aganglionic bowel was resected, so that these patients might have responded well to treatment without the major operation. Conservative management was attempted in the fourth patient, but the response was unsatisfactory: faeces had impacted in the upper third of his rectum at the age of 6 weeks leaving the ampulla empty. The aganglionic segment appeared unduly long for a successful 'PEARM' operation, but this was performed as a test. The diagnosis was confirmed, but the symptoms persisted until a sigmoid colostomy was established. There were no complications at the anastomosis at the subsequent successful Swenson's operation.

Group (c). Abnormal myenteric nerve with few ganglion cells. The 5 patients in this group were all boys who had a similar abnormality in the myenteric plexus just proximal to the internal anal sphincter. The nerve fibres appeared to lack myelin as in Hirschsprung's disease, but after searching several sections occasional ganglion cells could be found. Specimens were also taken from the distal valve of Houston in 2 patients who were identical twins, but the innervation was normal at this higher level. The symptoms started between the ages of 2 and 7 years in this group, and in 3 of the boys overflow of faeces resulted in chronic soiling. After operation, all these children had a variable period of anal incontinence lasting as long as several weeks, but all except one have slowly become clean and continent, though they need the occasional aid of small quantities of evacuants to empty their bowels. Symptoms persist in one of the twins, laxatives give him diarrhoea, and he is intolerant of suppositories. Psychiatric examination has revealed evidence of stress which occurred when he was tragically orphaned, and the symptoms are manifest at a point of organic weakness. His constipation has improved following left hemicolectomy with anterior restorative resection of the rectum, but a tendency to slight soiling persists.

It is notoriously difficult to assess the results of treatment for intractable constipation. Where an operation contributes both to diagnosis and treatment, any control observations become restricted to the earlier progress of the patients themselves and to general experience of similar cases. The 'PEARM' operation can assist in identifying patients with abnormalities in the intrinsic innervation of the distal rectum, and it may mark the turning point in the response to evacuants in selected cases. There can be dramatic relief of constipation after the operation and the use of evacuants can often cease, particularly in patients with ultra-short segment Hirschsprung's disease. The need for some patients to continue to take small quantities of laxatives is a trifling disadvantage of the minor operation compared to the alternative of a major operation.

Ultra-short segment Hirschsprung's disease is one cause of the syndrome of 'anal achalasia' (Hurst, 1934). The treatment originally advocated comprised the passage of a large anal dilator daily and the judicious use of laxatives. When classical Hirschsprung's disease is treated by restorative anterior resection of the rectum, a short proctodeal segment of aganglionic bowel remains and residual symptoms of constipation respond to anorectal dilatation (Rehbein and von Zimmermann, 1960). Dilatation has been found less effective than myotomy for the treatment of achalasia of the cardia. Myotomy of the internal anal sphincter can reduce the high anal tone which can persist and precipitate diarrhoea after restorative rectosigmoidectomy for Hirschsprung's disease (Swenson, Fisher, and Scott, 1960), and an extended ano-rectal myotomy combined with diagnostic rectal biopsy has recently been advocated as the treatment of choice for ultra-short segment Hirschsprung's disease (Bentley, 1962, 1964; Roviralta, 1962).

It seems probable that anal achalasia can have a variety of underlying causes which could include immaturity or degeneration of the intrinsic nerve in the distal rectum. Alternatively, local abnormalities, such as those in Group (c), could be due to a variant of ultrashort segment Hirschsprung's disease, with the transition towards aganglionosis starting just above the anal canal.

The cause of the symptoms is highly speculative when no histological abnormality is seen in the bowel wall. Difficulties arise in deciding whether psychological disturbances are the cause or the effect of faecal and urinary retention and overflow. Removal of infection and mechanical impediments does not always restore the ability of the dilated bowel or bladder to empty, and the evident impairment of the reflexes of evacuation is then unexplained. There is increasing evidence that such reflex activity is modulated in a subtle way by a variety of substances that are naturally found or formed in the affected tissues, and it is conceivable that their action could be disordered, possibly because of an inborn error. It is difficult to investigate this hypothesis as few of those affected die or undergo resection of the organs involved, but a better insight into some of the factors responsible for chronic constipation may be obtained, as knowledge of neurochemistry and smooth muscle activity increases and new techniques are developed that can be applied to study tissue excised from the rectal wall by the PEARM operation.

Summary. It is not always easy to distinguish the cause of intractable accumulation of faeces in the rectum. This can result from anal achalasia due to ultra-short segment Hirschsprung's disease. Experience with the minor operation of posterior excisional ano-rectal myotomy suggests that it can be helpful for both diagnosis and treatment, particularly when anal achalasia occurs with ultra-short segment Hirschsprung's disease. Some patients were found to have abnormalities of the intrinsic nerve differing from those in Hirschsprung's disease, because of the sparse occurrence of ganglion cells, but other patients did not demonstrate any histological abnormality. The significance of these findings is discussed.

\section{What is Pseudo-Hirschsprung's Disease? \\ H. H. NixoN}

Pseudo-Hirschsprung's disease is considered an unsatisfactory label because several different conditions 
imitate some of the characteristics of Hirschsprung's disease but none imitate them all. I would like to comment on 8 of these, finishing with the interesting group Ehrenpreis has described.

(1) Megarectum, Terminal Reservoir, or Rectal Inertia. Ravitch has called this pseudo-Hirschsprung's disease, but Table II shows how easily a typical case can be distinguished. Megacolon or colonic inertia are alternative names which are not so accurate, because the disorder involves the terminal bowel and the colon above remains remarkably normal in function. 'Secondary' or

TABLE II

Differentiation of Hirschsprung's Disease and Rectal Inertia

\begin{tabular}{|c|c|c|c|c|}
\hline & & & $\begin{array}{c}\text { Hirschsprung's } \\
\text { Disease }\end{array}$ & $\begin{array}{c}\text { Rectal Inertia or } \\
\text { Megarectum }\end{array}$ \\
\hline $\begin{array}{l}\text { Onset . } \\
\text { Constipation } \\
\text { Distension } \\
\text { Rectum . . } \\
\text { Soiling . } \\
\text { General health } \\
\text { Risk to life }\end{array}$ & $\begin{array}{l}\cdots \\
\cdots \\
\cdots \\
\cdots \\
\cdots\end{array}$ & \begin{tabular}{l|}
$\ldots$ \\
$\ldots$ \\
$\cdots$ \\
$\cdots$ \\
$\cdots$
\end{tabular} & $\begin{array}{c}\text { Neonatal } \\
+ \\
+ \\
\text { Empty } \\
- \\
\text { Poor } \\
\text { High }\end{array}$ & $\begin{array}{c}\text { Training period } \\
+ \\
- \\
\text { Loaded } \\
+ \\
\text { Reasonable } \\
\text { Negligible }\end{array}$ \\
\hline
\end{tabular}

'symptomatic' megacolon are also inappropriate names, because true Hirschsprung's disease is itself a megacolon secondary to, or symptomatic of, the distal aganglionic bowel. The same anatomical and physiological findings arise in those secondary to anal stenosis, as in those of functional (or 'idiopathic') origin (Callaghan and Nixon, 1964). The basic fault seems to be 'persistent failure to respond to the call to stool' (Hurst, 1925) for physical or psychological reasons.

A few neglected cases come to imitate the clinical picture of Hirschsprung's disease, but the early history is different and rectal biopsy differentiates the two conditions.

(2) Congenital Constipation. Coekin and Gairdner (1960) have drawn attention to a group clinically like the above terminal reservoir group but having an earlier onset in the newborn period. Harris, Corbin, and Hill (1954) have suggested that these may result from minor anal stenosis which relents as a result of rectal examination and/or the passage of stools. Two of our cases of this nature had a difficult birth and may have had a cerebral influence on the motility of the bowel in the early days of life.

(3) Immaturity. One premature baby presented after birth with typical signs and symptoms of Hirschsprung's disease and required rectal wash-outs. A rectal biopsy showed ganglion cells, but Dr. Crome thought they looked abnormal: perhaps these were immature cells as will be described by Spencer. At all events the condition settled in a few weeks and the baby is now well. Similar resolving symptoms have been seen in other babies, not always very premature.

(4) Meconium Plug. This syndrome, well described by Zachary (1957) and Clatworthy, Howard, and Lloyd (1956), imitates the neonatal signs and symptoms of Hirschsprung's disease, but there is no further trouble after passage or evacuation of the plug.

(5) Faecal Plug. This syndrome presents a few days after birth as an obstruction imitating Hirschsprung's disease, but some meconium is usually passed before this becomes obvious (Zachary, 1957). Hard concretions block the bowel and may lead to perforation-even below a good colostomy, as in one of our cases. The concretions should therefore be removed at the laparotomy.

(6) Segmental Megacolon. Swenson and Rathauser (1959) described localized dilatation and hypertrophy of segments of colon without evident cause and with normal ganglia. We have had one similar localized enlargement in the terminal ileum.

(7) Acquired Hirschsprung's Disease. Ehrenpreis (1965b) has described a case apparently due to ischaemic atrophy of the ganglia in the bowel mobilized for a pullthrough operation. We have seen a baby who developed abdominal distension, constipation, and vomiting after an abdomino-anal pull-through operation for rectal agenesis. The left colon had been mobilized with division of the inferior mesenteric vessels at their origin; at re-operation 3 weeks later it was congested and blue, though viable, and appeared to represent an earlier stage of the same process. Perhaps lesser degrees of this change may account for some other poor results of pullthrough operations.

The acquired megacolon due to Chagas' disease with its destruction of ganglia is now well known. It imitates Hirschsprung's disease but occurs in an older age-group.

(8) Adynamic Ileus with Dilatation. We have had 3 cases that seem similar to those described as pseudoHirschsprung's disease by Ehrenpreis (1965a) and Bill, Creighton, and Stevenson (1957). There was constipation with distension and bouts of vomiting from birth. There was, however, complete absence of hypertrophy in the dilated bowel. In our cases the most striking difference from Hirschsprung's disease was the absence of peristalsis. The abdomen was silent and action could not be stimulated-this is the complete opposite of the overacting colon of the aganglionic disease, and the application of a stethoscope to the abdomen would differentiate the conditions. The inactivity appeared to involve the entire intestine.

Two of our cases died of persistent amotility, one with a transverse colostomy and the other with an ileostomy. Balloons inserted into the bowel registered no pressure changes. Wash-outs produced little evacuation. Carbachol, and in one case ismelin and spinal anaesthesia were also ineffective. Histology revealed normal ganglia and villi. The electrocardiogram and serum potassium, magnesium, and calcium were normal. Urinary excretion of HMMA was normal. Histochemical examinations were unsatisfactory. The brain weights were low-one was $520 \mathrm{~g}$. (oedematous and perhaps truly only about $420 \mathrm{~g}$.), when the average for the age was 590 g., and the other $850 \mathrm{~g}$. compared with an average for the age of $1,042 \mathrm{~g}$. The head circumferences were low by more than 4 standard deviations and 2 standard deviations, respectively, the latter baby having had a high birth weight of $4,488 \mathrm{~g}$. On examination the brains had small gyri with wide sulci. The late Dr. M. Bodian suggested that there might perhaps have been some central influence on the bowel activity. 
Our third case began similarly but after a few months relented. The previously ineffective transverse colostomy began to discharge frequent loose stools. It was closed and a normal bowel habit was established. The child remains well 3 years later.

Perhaps histochemical studies will elucidate the aetiology of this unexplained adynamic group of which the cardinal sign appears to be the silent abdomen.

\section{Problems in Rectal Biopsy due to Immaturity of Ganglion Cells}

\section{B. SPENCER}

[Dr. Spencer emphasized that the observations he was presenting were derived from Dr. Blanca Smith but that he had confirmed the observations in two patients.] At present it is not usual to take account of the age of the patient when a search is made for ganglion cells on rectal biopsy, as the differences in the morphology that occur with maturation have not been anticipated. The changes that take place in neurones developing in the central nervous system have been well established. The apolar neuroblast gradually progresses to the multipolar neurone. This pattern has also been noted in the neurones of the autonomic ganglia. Similar changes are possible in the ganglion cells of the rectum, and failure to recognize the immature ganglion cells could lead to an erroneous diagnosis of aganglionosis. Dr. Blanca Smith has studied material from 30 necropsies, ranging in age from embryos to children of 5 years. Specimens were taken from the cardia, the pylorus, and the last $10 \mathrm{~cm}$. of bowel. All the embryos had immature neuroblasts in the rectum, in the premature infants most of the rectal ganglion cells were mono- or bipolar. Few immature ganglia were present in corresponding sections taken from full-term infants, and all the neurones were of adult type in children of 2 to 5 years. Thus the ganglion cells of the rectum may not be completely differentiated at birth and few are multipolar. Their appearance is determined more by the length of the pregnancy than the birthweight of the infant. Maturation appears to progress in a cephalo-caudad direction, and the myenteric (Auerbach's) plexus matures several weeks earlier than the submucous (Meissner's) plexus.

Paediatric surgeons should undertake the management of chronic constipation in infants and children, even when no organic disease can be demonstrated. The surgeon probably has the clearest understanding of the mechanical aspects of the problem, and he should learn more of the problems of selecting appropriate medication and rectifying any associated psychological disturbance. At present there is a grave danger that these unfortunate children will 'drop into the interspeciality crevice', and so drift from physician to physician until they are irretrievably lost.

\section{Abnormal Arteries in Hirschsprung's Disease JAMES LISTER}

When Swenson and Bill (1948) renewed interest in the unexpanded distal segment of colon, and Bodian, Stephens, and Ward (1949) confirmed the absence of ganglion cells in this segment in all cases of Hirschsprung's disease, it seemed that 60 years of confusion in definition and diagnosis had come to an end. But, as Nixon has said, more refined studies of the myenteric plexuses reveal many variations of the basic congenital abnormalities, and indeed abnormalities are not necessarily confined to nerve cells, so that precise diagnosis once more becomes very difficult.

If we accept that the clinical picture of Hirschsprung's disease is brought about by the failure of propulsion across a segment of colon, then we should be prepared to accept that there are several possible causes for the failure of that contraction. These causes can be divided into two groups-those in which the myenteric plexus is normal and those in which it is abnormal. The first group includes those cases in which there appears to be a central paralysis, such as in cerebral atrophy (Nixon, 1961) or myxoedema (Ravitch, 1958), and those in which a normal plexus is paralysed peripherally, such as in the ileus associated with sepsis, or other toxins; this type of toxic paralysis can lead to cases in the second group where there is residual histological abnormality with degeneration and perhaps disappearance of ganglion cells as in Chagas' disease (Ferreira-Santos, 1961).

In the second group of causes the myenteric plexus is primarily abnormal at birth, either with small immature ganglion cells, apparently not reached by a preganglionic fibre, or with dysplasia, sometimes confined to the neural elements but sometimes involving vascular elements as well, or perhaps involving vascular elements alone.

Attention was drawn to abnormalities in arteries when, at operation, vessels were seen running in the mesentery of the colon, most conspicuously in the junctional zone between expanded and unexpanded bowel, vessels which were firm and thickened, looking rather like an inflammatory peri-arteritis. On section, however, they presented the features of vessels in a vascular hamartoma with a completely bizarre appearance, sometimes with bunched-up muscle fibres and sometimes with an obliterative endarteritis.

This vascular abnormality has been seen in 3 out of the last 10 cases of histologically proven Hirschsprung's disease, one at the age of 18 months, and 2 specimens obtained in the neonatal period. The significance is perhaps a little obscure; do these vessels indicate a poor foetal blood supply which leads to degeneration of the myenteric plexus in utero? Or is the vascular dysplasia the result of the same insult that produced the neuronal dysplasia?

It is particularly interesting that one specimen of these abnormal vessels was obtained from a $1 \cdot 2 \mathrm{~kg}$. premature baby who had a colostomy made at 5 days for a large bowel obstruction thought to be Hirschsprung's disease; he had a rectal biopsy taken at 15 months to confirm the diagnosis, and this showed a normal myenteric plexus. The colostomy was closed and 6 months later he was doing well. Was he another case of pseudo-Hirschsprung's disease? 


\section{Histological Investigations into 'Idiopathic Megacolon'}

\section{BERNARD DUHAMEL}

Megacolon in infants and children is usually considered in two main groups (Bodian et al., 1949). Congenital megacolon (Hirschsprung's disease) is clearly associated with an abnormal nerve structure in the bowel wall; when this abnormality cannot be demonstrated the megacolon is deemed to be functional. Relative narrowing of the distal bowel is seldom encountered with 'functional megacolon', indeed the rectum is often distended and the appearances are consistent with the concept of 'anal achalasia' (Hurst, 1934). Functional megacolon may be acquired in childhood because of psychological disturbances or faulty diet, and is then responsive to appropriate treatment, but when the trouble is manifest from birth or weaning, medicinal, dietetic, and psychological treatment seem of little value, and the term 'idiopathic megacolon' is then applied.

Rectal biopsy performed on patients with idiopathic megacolon using the method devised by Swenson, Fisher, and MacMahon (1955) yields a small piece of rectal wall with normal intrinsic nerve tissue, but if a specimen that is fully representative of the ano-rectal wall is examined abnormal tissues will be disclosed in some of these patients.

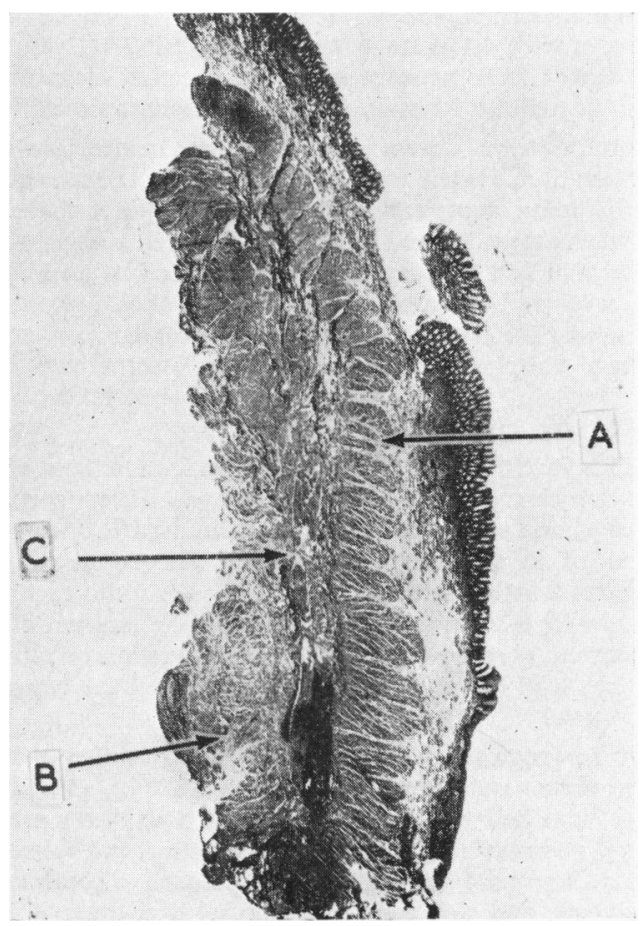

FIG. 3.-Sagittal section of ano-rectal wall ( $\times$ 5.) A. Internal anal sphincter. B. External anal sphincter. $C$. Longitudinal muscle of the rectum.
The histology of the bowel wall in the vicinity of the internal anal sphincter can be difficult to interpret, and there is still some debate regarding the distribution of ganglion cells at the level of the human internal anal sphincter. Few observations have been recorded, and on this scanty evidence the contrary allegations have been made that ganglion cells are not normally found in the vicinity of the internal anal sphincter and that ganglion cells are invariably present in this situation, even in patients with Hirschsprung's disease. In order to determine the normal distribution of the intrinsic ganglion cells in this area, I have examined the ano-rectal region of 100 dead infants and children in collaboration with Dr. Roujeau (Pathologist, Hôpital de St. Denis, Paris). The specimens included the whole length of the internal anal sphincter and the distal rectal wall, they were obtained from stillborn infants and children whose cause of death was unconnected with the bowel. In $25 \%$ ganglion cells were present in a nerve plexus situated between the internal and external anal sphincters. This nerve plexus appears to be derived from the rectum, as it was only present where the longitudinal muscle of the rectum extended down between the internal and external anal sphincters (Fig. 3). This was mostly seen in specimens from older children, and was uncommon in the newborn.

Comparable representative specimens for histological examination were obtained from living patients with megacolon. A strip of bowel wall was excised from the anal canal and distal rectum as follows: (i) The anus is dilated and a speculum is inserted. (ii) The mucocutaneous junction is tensed and incised between two forceps that grasp the anal verge. (iii) The mucosa is raised off the underlying muscle and the cleft between the internal and external anal sphincters is defined. (iv) A strip of submucous tissue including a segment of the internal anal sphincter is excised with scissors. The specimen is $5 \mathrm{~cm}$. long and $1 \mathrm{~cm}$. wide (Fig. $4 \mathrm{a}$ and b). (v) Interrupted silk sutures are used to close the incision and a pack in the anal lumen secures haemostasis.

This operation is quick, simple, and free of complications. It has a therapeutic effect in anal achalasia comparable to the Fredet-Ramstedt operation for hypertrophic pyloric stenosis, or Heller's operation for achalasia of the cardia. It has been performed on 21 children with intractable symptoms attributed to idiopathic megacolon. The muscle and nerve tissue appeared normal in only three specimens. In 8 cases, the myenteric nerve was normal, but the structure of the internal sphincter was abnormal. In some cases fibrosis of variable extent involved and bound together the striated and unstriated muscle of the anal sphincter; in other cases there was even a complete absence of unstriated muscle, and the internal sphincter was a striated muscle. The anomalies were the same as in congenital stenosis or ectopic micro-anus. These patients appeared to have minor variations of the group of ano-rectal anomalies classed as 'caudal regression', as demonstrated by frequent association in these cases of associated vertebral or genito-urinary malformations, and in a number of cases, the anal canal was situated further 


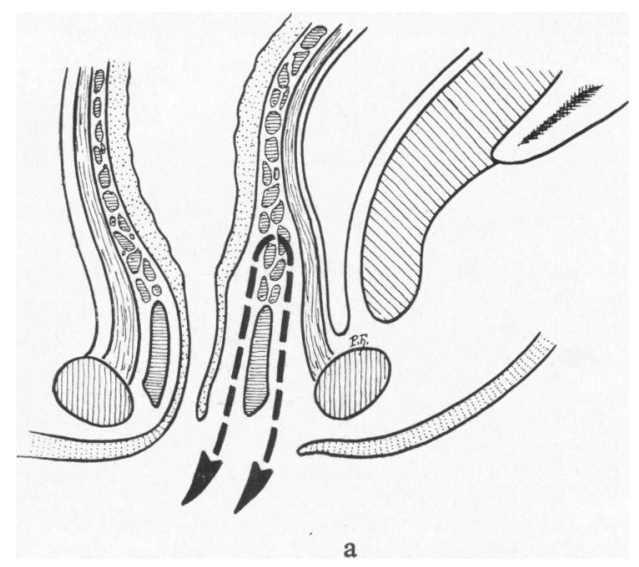

FIG. 4 a and b.-Submucous sphincterectomy (see text).

forward than usual. The remaining 10 patients had histological appearances of ultra-short segment Hirschsprung's disease (Bentley, 1964). The specimen did not always include the upper limit of aganglionosis which was evident in the inferior part of the rectal wall. In these patients, the clinical and anatomical picture resembled that seen following inadequate distal resection of the rectum in connexion with recto-sigmoidectomy for Hirschsprung's disease. Two of these patients did not improve significantly after the extended internal anal sphincterotomy, and they were treated by a retro-rectal pull-through resection and anastomosis.

The foregoing observations suggest that great caution is required in arriving at the diagnosis of idiopathic megacolon. Fully representative biopsy of the anorectal wall is essential to exclude ultra-short segment Hirschsprung's disease or fibrosis of the anal sphincters. Fortunately biopsy by the technique described involves an extended internal anal sphincterotomy that assists in relieving the symptoms when organic disease is demonstrated.

\section{Intrinsic Non-propulsive Colon}

RoBert PAgÈs and BERNARd DUHAMEL

Intrinsic failure of colonic propulsion is a rare cause of intestinal obstruction in infancy and childhood. 13 patients were seen at the Hôpital de St. Denis (Paris). The clinical picture was always compatible with extensive colonic aganglionosis, but this anomaly was not invariably present to account for the impaired activity. Some of the patients presented as neonatal intestinal obstruction; in others obstruction developed in early infancy, either acutely or after a period of progressive constipation, abdominal distension, and vomiting. Radiographic examination demonstrated distension of the small intestine but no distension of either the whole or the distal half of the colon. An opaque enema occasionally demonstrated proximal dilatation of the colon, but

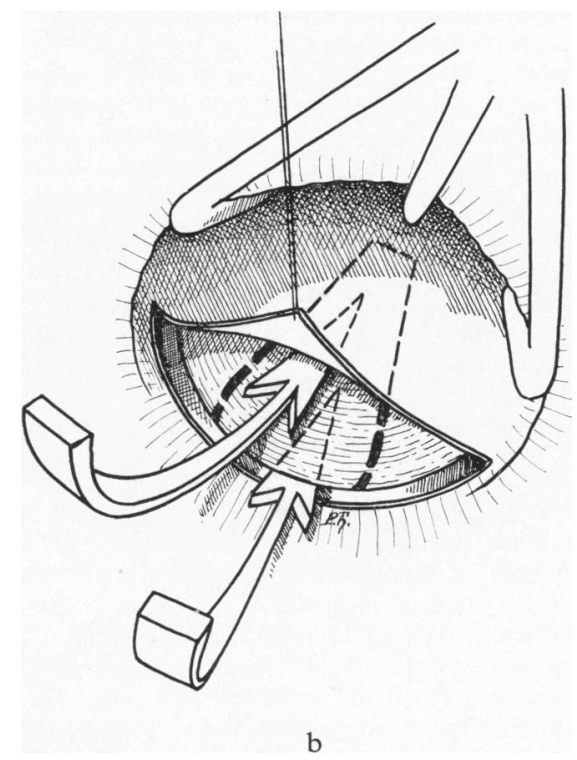

even where there was no evidence of colonic distension the contrast medium failed to be evacuated fully in 24 hours.

Subsequent histological examination of the colon was based on specimens removed at operation or necropsy. 9 patients demonstrated some abnormality in the intrinsic nerves, but no abnormality could be detected in 4. 3 patients had aganglionosis of the submucous and myenteric plexuses, with thick bundles of non-myelinated nerve fibres as seen in Hirschsprung's disease. These abnormalities affected the entire colon in 2 patients, and in the third rather more than the distal half of the colon was involved. The remaining 6 demonstrated abnormalities of the submucous or myenteric plexus that were atypical for Hirschsprung's disease. In one of these patients the appearances suggested Hirschsprung's disease with a transitional zone extending to $20 \mathrm{~cm}$. Two patients had limited zonal aganglionosis with normal innervation above and below the aganglionic ring, and one patient had absent ganglion cells but normally-formed intrinsic nerve fibres. In 3 the aganglionosis involved either the submucous or the myenteric plexus, but not both, and in 2 the ganglion cells were present, though scanty.

The gross morbid anatomy was dictated by the extent of the impairment in propulsive activity rather than by the histological findings. 6 patients had impairment of propulsion affecting the entire colon, with consequent gross distension of the small intestine. 5 of them died, 1 recovered following retro-rectal trans-anal ileostomy, but only 3 were fit for such operative intervention. In 7 patients the defect in propulsion affected rather more than the left half of the colon. The small intestine and the right half of the colon were distended. All were treated by retro-rectal trans-anal anastomosis, the right colon being turned down into the pelvis for anastomosis. 4 of these patients recovered and 3 died. 
The consequences of extensive intrinsic impairment of colonic propulsion are grave. In this series 8 patients died and only 5 survived following an extensive operation, despite the fact that proctocolectomy was avoided. The practical management of these patients should be determined by the extent of the functional failure, and not exclusively by the extent of any histological abnormality in the intrinsic innervation. In consequence we propose to use the term intrinsic non-propulsive colon to describe this syndrome, rather than to designate some of the examples as long-segment Hirschsprung's disease. Indeed it is debatable whether the term Hirschsprung's disease can be applied in the absence of megacolon, since this was one of the features that was originally emphasized by Hirschsprung. It is true that the megacolon is a secondary development, but following the acceptance of the underlying pathology of a distal aganglionic segment as a result of the convincing demonstration by Bodian et al. (1949) of the abnormal intrinsic neurohistology, there has been a regrettable tendency to equate aganglionosis with Hirschsprung's disease. Indeed, it is now evident that Hirschsprung's disease, as originally described on clinical grounds, can occur without the typical abnormalities in the intrinsic nerve tissue of the distal bowel, and many examples have been reported (Roviralta and Casasa, 1962). We can add one case of rectosigmoidal 'Hirschsprung's disease' from our own experience, and we suggest that these cases, otherwise designated as 'pseudo-Hirschsprung's disease', could well be included as shorter segment examples of the intrinsic non-propulsive colon syndrome. Indeed, Hirschsprung's disease with typical aganglionosis is also a manifestation of the syndrome. Rectal and colonic antiperistalsis, first demonstrated by Roith (1913), can also impede effective colonic propulsion when the polarity of action is persistently reversed. We have had 2 examples, in one a preliminary colostomy, which was not closed at the time of retro-rectal trans-anal anastomosis, continued to act and anal defaecation did not occur; in the other the blind colonic loop formed a fistula with the stomach that could be demonstrated by an opaque enema but not by swallowed barium. We have also seen 2 patients in whom the zone of impaired propulsion became more extensive with the passage of time. Symptoms recurred some time after a Duhamel procedure for relatively short segment malfunction, and at re-operation the right colon and the ileum respectively had to be used for the trans-anal anastomosis.

To summarize, intestinal obstruction in infancy and childhood can result from an intrinsic failure of colonic propulsion. It is rare for this to involve the greater part of the colon, but 13 such cases are described. Most, but not all of these, were associated with abnormalities in the intrinsic nerve supply of the bowel, and where there was distension of the colon this was limited to the proximal gut. There are grounds for including classical Hirschsprung's disease as a 'short segment' variant of this syndrome, and it is emphasized that rare examples of clinical Hirschsprung's disease occur without histological evidence of distal aganglionic bowel.

\section{Pseudo-Hirschsprung's Disease in Bantu Children}

\section{ARNold Katz}

The final arbiter in the diagnosis of Hirschsprung's disease is the identification of aganglionosis on adequate rectal biopsy. A rectal biopsy is essential for diagnosis, as Hirschsprung's disease can be simulated by other conditions. A series of 68 cases of Hirschsprung's disease has been seen at the Red Cross War Memorial Children's Hospital in Cape Town, 24 in white children, 36 in coloured patients, and 8 in Bantus. These figures suggest that Hirschsprung's disease is rare amongst Bantu children. Amongst the Bantu children referred to the hospital were 4 patients who were at first thought to have Hirschsprung's disease, and the first 2 patients were treated as such. In these children the duration of chronic constipation and abdominal distension was difficult to determine. On examination there was marked gaseous distension of the abdomen and the rectum was empty of faeces. Proximal dilatation of the colon was evident at the recto-sigmoid junction on contrast radiography and on laparotomy, but the dilated colon was thin walled, without evidence of hypertrophy. Histological examination revealed atrophy of both the muscle layers of the colon and rectum, but this was most striking in the dilated bowel where the sections of the muscle appeared beaded due to alternating short zones of atrophy with the intervening muscle tissue being slightly hypertrophied. There was mild infiltration of the muscle layers with lymphocytes, eosinophils and mast cells, together with an increase in interstitial collagen. The ganglion cells were plentiful and normal. The first patient was treated by Swenson's operation without preliminary rectal biopsy. $\mathrm{He}$ was subsequently admitted elsewhere with duodenal ileus, and laparotomy was carried out. Further histological investigations demonstrated inflammation and atrophy in the muscle of the duodenum, jejunum, and throughout the colon. These changes were also present in the 3 other cases that were treated by sigmoid resection, Duhamel's operation, and exploratory laparotomy.

The symptoms in these 4 patients did not start from birth and rectal biopsy in 2 patients revealed normal ganglion cells. The remote possibility of a variant of Chagas' disease was excluded, as ganglion cells were plentiful at all levels of the intestines, and muscular inflammation and atrophy is not a feature of Chagas' disease. Trypanosomiasis is not encountered in Southern Africa apart from Trypanasomiasis rhodesianensi which causes sleeping sickness (mostly in central Africa). The distal rectum in these patients showed no abnormality in morphology, activity, or histology, and the possibility arises that they were all victims of the same, hitherto undescribed disease.

\section{Discussion}

J. F. R. Bentley emphasized that current knowledge of the motor functions of the colon and rectum remained incomplete. Why does a normal healthy foetus not have bowel actions in utero? What is the inhibiting factor and what triggers the onset of defaecation at birth ? What 
regulates the rate and force of colonic propulsion in a normal person? What prevents us from having continuous diarrhoea? Is propulsive activity the same as peristalsis, where the wave of contraction is preceded by a wave of relaxation? This does not seem to occur in the ureter and it may not be essential in the gut. Indeed myogenic propulsive activity in humans may not be identical with that in laboratory animals, as patients with extensive colonic aganglionosis do not necessarily have early acute intestinal obstruction and some of them continue to have bowel actions for several months. The role of naturally occurring substances with a profound effect on the activity of the smooth muscle of the bowel has yet to be elucidated. These substances include 5-hydroxytryptamine (and its antagonists), excessive production of which can occur with carcinoid tumours, the patient suffering from diarrhoea and a degree of megacolon. Megasigmoid and volvulus is common in Africans who live on bananas which have a high $5 \mathrm{HT}$ content. The most potent agent is the polypeptide substance $P$ which has not been extracted in a pure form, despite its extreme lability. It occurs near the ganglion cells mostly in the muscularis mucosae. Biological activity in tumours such as neuroblastomas is attributed to catecholamines, and these tumours too can be associated with diarrhoea and dilatation of the colon. Other agents include bradykinin, villikinin, kallidin, cholecystokinin, darmstoff of Vogt, and GABA ( $\gamma$-aminobutyric acid). Finally, the precise mode of action on the bowel of the oldest drugs, the purgatives and opium, is still a mystery.

$J . J$. Shepherd outlined investigations he had made on the response of the human colon to stimulation with a variety of agents. He concluded that in Hirchhsprung's disease the sympathetic innervation was deficient, and that this was the cause of the 'contracted segment'. The absence of ganglion cells does not account for the 'contracted segment', indeed the diminution of ganglion cells in Chagas' disease is associated with dilatation of the bowel. He suggested that the absence of ganglion cells was not the dominant factor in the pathology of Hirschsprung's disease, and that pseudo-Hirschsprung's disease could be the result of sympathetic deficiency in circumstances where the ganglion cells were present. Indeed, in Hirschsprung's disease there was hypersensitivity of the aganglionic segment to adrenaline, and a diminished sensitivity to acetylcholine, consistent with absence of sympathetic innervation.

$A$. Katz observed that patients who had been subjected to bilateral sympathectomy often developed meteorism. He had also noticed that patients with total colectomy and ileo-anal anastomosis had less frequent bowel actions when given a diet of bananas.

F. Rehbein emphasized that where there was a chronic accumulation of faeces in the rectum, there were two factors to consider: one was the megarectum and the consequences of chronic dilatation on its activity, the other was the state of the anal sphincter. Where the dilatation of the bowel was so great that recovery of activity was improbable it should be excised, but where the impediment to evacuation was anal achalasia this could respond to forceful dilatation. He had employed anal dilatation in this way as adjunct to the treatment of Hirschsprung's disease by anterior restorative resection of the rectum and sigmoid with very satisfactory results.

J.F. R. Bentley recalled that Sir Arthur Hurst had first suggested forceful anal dilatation for the treatment of anal achalasia.

I. P. Todd agreed that posterior excisional ano-rectal myotomy could be a good operation in selected cases of megarectum, but one of his adult patients had been made incontinent. He emphasized that some patients with megarectum had anal sphincters that did not relax when the rectum was further distended, and that these patients could be expected to do well with sphincterotomy.

H. B. Lynn recounted his experience with neurofibromatosis of the intestinal tract associated with magacolon and chronic constipation. The myenteric plexus accounted for one-third of the thickness of the bowel wall in specimens from the rectum, sigmoid, appendix, ileum, and jejunum. He had performed a type of excisional myotomy on 2 patients with short segment Hirschsprung's disease, who were only in hospital for one night. Their parents were delighted to have normal children the next day. He had also performed the operation on 3 patients who had been treated by anterior resection of the rectum and sigmoid for Hirschsprung's disease, with complete relief of symptoms. In 2 patients with persisting constipation following Swenson's operation, one had had tremendous relief, but the other, his own patient, was no better.

B. Morson commented on Prof. Duhamel's histological observations. His own experience was derived from adults, but he had never seen striated muscle fibres mixed up with smooth muscle in the internal anal sphincter.

$T . M c C o l l$ discussed the ano-rectal musculature in view of Prof. Duhamel's observations. In about 50 specimens examined the longitudinal muscle layer had almost always extended nearly as far as the lower border of the internal sphincter.

H. H. Nixon considered that some of the patients encountered by Prof. Duhamel might have been suffering from minor degrees of ano-rectal abnormality.

M. M. Ravitch who coined the term 'pseudoHirschsprung's disease' in 1958, discussed nomenclature. He admitted that it was not a very good term but emphasized that he did not like to use the term 'idiopathic' which had no meaning and should be banished. If we mean that 'we don't know the cause', we probably ought to say 'agnogenic'. With regard to the condition of megacolon with a long distal narrow segment containing ganglion cells, he proposed the use of the name Ehrenpreis disease. 


\section{REFERENCES}

Bentley, J. R. F. (1962). Posterior partial proctectomy in the diagnosis and treatment of ano-rectal aganglionosis. Exhibition Catalogue, 10th int. Congr. Pediat., Lisbon.

- (1964). Some new observations on megacolon in infancy and childhood with special reference to the management of megasigmoid and megarectum. Dis. Colon Rect., 7, 462.

Bill, A. H., Jr., Creighton, S. A., and Stevenson, J. K. (1957). The selection of infants and children for the surgical treatment of Hirschsprung's disease. Surg. Gynec. Obstet., 104, 151.

Bodian, M., Stephens, F. D., and Ward, B. C. H. (1949). Hirschsprung's disease and idiopathic megacolon. Lancet, 1, 6.

Callaghan, R. P., and Nixon, H. H. (1964). Megarectum: physiological observations. Arch. Dis. Childh., 39, 153.

Clatworthy, H. W., Jr., Howard, W. H. R., and Lloyd, J. (1956). The meconium plug syndrome. Surgery, 39, 131.

Coekin, M., and Gairdner, D. (1960). Faecal incontinence in children. The physical factor. Brit. med. F., 2, 1175.

Davidson, M., and Bauer, C. H. (1958). Studies of distal colonic motility in children. IV. Achalasia of the distal rectal segment despite presence of ganglia in the myenteric plexuses of this area. Pediatrics, 21, 746.

Ehrenpreis, Th. (1965a). Pseudo-Hirschsprung's disease. Arch. Dis. Childh., 40, 177.

- (1965b). Acquired megacolon as a complication of rectosigmoidectomy for Hirschsprung's disease. ibid., 40, 180.

Ferreira-Santos, R. (1961). Megacolon and megarectum in Chagas' disease. Proc. roy. Soc. Med., 54, 1047.

Harris, L. E., Corbin, H. P. F., and Hill, J. R. (1954). Anorectal rings in infancy. Pediatrics, 13, 59.
Hurst, A. F. (1925). The sphincters of the alimentary canal and their clinical significance. Brit. med. $\mathcal{F} ., 1,145$.

(1934). Anal achalasia and megacolon (Hirschsprung's disease; idiopathic dilatation of the colon). Guy's Hosp. Rep., 84, 317.

Nixon, H. H. (1961). In Discussion on megacolon and megaractum with emphasis on conditions other than Hirschsprung's disease. Proc. roy. Soc. Med., 54, 1037.

Ravitch, M. M. (1958). PseudoHirschsprung's disease. Ann. Surg., 147, 781 .

Rehbein, F., and von Zimmermann, H. (1960). Results with abdominal resection in Hirschsprung's disease. Arch. Dis. Childh., 35, 29.

Roith, D. (1913). Ueber die Peristaltik und Antiperistaltik des menschlichen Dickdarmes. Mitt. Grenzgeb. Med. Chir., 25, 203.

Roviralta, E. (1962). Nouvelles orientations chirurgicales dans le traitment du mégacôlon congénital. Ann. Chir. infant., 3, 155.

-, and Casasa, J. M. (1962). Le Mégacôlon Congenital, Diagnostic et Traitement (French adaptation by B. Duhamel and C. De Groote). Masson, Paris.

Swenson, O., and Bill, A. H., Jr. (1948). Resection of rectum and rectosigmoid with preservation of the sphincter for benign spastic lesions producing megacolon. Surgery, 24, 212.

- Fisher, J. H., and MacMahon, H. E. (1955). Rectal biopsy as an aid in the diagnosis of Hirschsprung's disease. New Engl. $\mathcal{F}$. Med., 253, 632.

$\longrightarrow,-$, and Scott, J. E. S. (1960). Diarrhoea following rectosigmoidectomy for Hirschsprung's disease. Surgery, 48, 419.

- and Rathauser, F. (1959). Segmental dilatation of the colon. Amer. F. Surg., 97, 734.

Zachary, R. B. (1957). Meconium and faecal plugs in the newborn. Arch. Dis. Childh., 32, 22. 